\title{
A Relation between Local and Total Energy in General Relativity
}

\author{
Gary T. Horowitz ${ }^{\star}$ and Paul Tod ${ }^{\star}$ \\ Mathematical Institute, 24-29 St. Giles, Oxford, England
}

\begin{abstract}
It is shown that, for an asymptotically flat space-time, there exists a collection of conserved vector fields which depend on the local stress energy of the matter and whose integrals over space-like hypersurfaces yield the total ADM or Bondi energy-momentum of the space-time. These vector fields can be used to prove the positivity of the ADM and Bondi energies.
\end{abstract}

\section{Introduction}

For an isolated system in general relativity, there are two distinct regimes where the concept of energy is well-defined: Locally, one has the energy density of matter fields given by the stress energy tensor $T_{a b}$; while asymptotically, far from the system, one can define a total energy of the system which is given by an energy momentum four vector $P^{a}$. It has been a long standing problem in general relativity to relate the asymptotically defined total energy to the energy density of matter in the interior. The reason that this problem arises at all, and turns out to be quite difficult to resolve, is that the asymptotic energy and the local energy are related in a complicated nonlinear way through Einstein's field equation. This issue is further complicated by the fact that the asymptotic energy includes contributions from the gravitational field as well as the matter, but there is no local energy density for the gravitational field analogous to $T_{a b}$.

Actually, there are two distinct notions of "total energy-momentum" for isolated systems. At large space-like separations from the source one can define the Arnowitt-Deser-Misner (ADM) four momentum $P_{a}^{\text {ADM }}[1]$, and at large null separations one can define the Bondi four momentum $P_{a}^{\mathrm{B}}[2]$. The main difference between the two is that the Bondi momentum is dynamical while the ADM momentum is not, i.e. the Bondi momentum is associated with an instant of retarded time and changes according to the radiation which the source emits,

* NATO Postdoctoral fellow. Current address: Institute for Advanced Study, Princeton, NJ 08540, USA

$\star \star$ S.R.C. Research assistant 
while the ADM momentum is a fixed vector given once and for all. It is known that the Bondi energy decreases with time in the presence of radiation $[2,3]$, that is, radiation always carries away positive energy, and that under suitable conditions, the past limit of the Bondi momentum is the ADM momentum [4]. One would like to know how both $P_{a}^{\mathrm{ADM}}$ and $P_{a}^{\mathrm{B}}$ are related to the matter in the interior.

There exist well-known relations between local and total energy in two special cases. The first case is when the space-time admits a time-like Killing vector field $\xi^{a}$. In this case there is no radiation so $P_{a}^{\mathrm{B}}$ and $P_{a}^{\mathrm{ADM}}$ are equal and proportional to the limiting value of the Killing vector. The constant of proportionality, i.e. total energy $E$, is given by the Komar integral [5], which can be expressed as an integral over an asymptotically flat space-like three surface $\Sigma$ :

$$
E=\frac{1}{16 \pi} \int_{S} \varepsilon_{a b c d} \nabla^{c} \xi^{d} d S^{a b}=2 \int_{\Sigma}\left(T_{a b}-1 / 2 T g_{a b}\right) \xi^{a} d \Sigma^{b},
$$

where $S$ is an asymptotic two-sphere on $\Sigma$. The second case is when the gravitational field is weak. In this case there is e.g. an explicit expression for the Bondi energy as a retarded integral over the stress tensor [6].

In a recent paper [7] Witten gave a proof that $P_{a}^{\mathrm{ADM}}$ is a future-directed timelike or null vector if the matter satisfies a local positive energy condition, the dominant energy condition. (This theorem was first proved by Schoen and Yau [8].)

The essence of Witten's proof is the derivation of an expression relating $P_{a}^{\mathrm{ADM}}$ to $T_{a b}$ at one time, i.e. on one asymptotically flat space-like hypersurface. The purpose of this paper is to show that Witten's expression can be extended to yield a more general formula relating asymptotic energy to the local stress tensor $T_{a b}$. More precisely, we show that there exist conserved vector fields $p_{a}$ which act like "energy momentum densities" in the sense that

$$
\int_{\Sigma} p_{a} d \Sigma^{a}=P_{a}^{\mathrm{ADM}} V_{0}^{a}
$$

where $\Sigma$ is any asymptotically flat space-like three surface and $V_{0}^{a}$ is an asymptotic translation at spatial infinity. Further, for other choices of $p_{a}$,

$$
\int_{\Sigma^{\prime}} p_{a} d \Sigma^{a}=P_{a}^{\mathrm{B}} V_{0}^{a},
$$

where $\Sigma^{\prime}$ is an asymptotically null surface and $V_{0}^{a}$ is an asymptotic translation at null infinity.

The organisation of this paper is as follows. In Sect. 2 we review Witten's argument, obtaining his identity in the two-component spinor formalism. We then introduce the vector fields described above as conserved vectors associated with solutions of the Weyl neutrino equation. We next show that by choosing these solutions to satisfy the appropriate boundary conditions, we can relate the conserved vector fields to $P_{a}^{\mathrm{ADM}}$ and $P_{a}^{\mathrm{B}}$.

In Sect. 3 we consider the boundary conditions at null infinity in more detail and show that solutions to the Weyl equation with these boundary conditions 
indeed exist. Applications of these vector fields to positive energy theorems and other issues are discussed in the conclusion ${ }^{1}$.

\section{The Relation between Local and Total Energy}

We begin by reviewing Witten's proof of the positive energy conjecture at spatial infinity. This will serve the dual role of familiarising the reader with our notation and introducing Witten's expression which we will then generalise.

Let $\left(M, g_{a b}\right)$ be a space-time with a nonsingular, asymptotically flat, space-like three surface $\Sigma$. Suppose the dominant energy condition holds on $\Sigma$, i.e. $T_{a b} t^{a}$ is a future directed time-like vector for any future directed time-like $t^{a}$. We wish to show, following Witten, that $P_{a}^{\mathrm{ADM}}$ is a future directed time-like or null vector.

Let $t^{a}$ be the unit normal to $\Sigma$, let ${ }^{2} h_{a b}=g_{a b}-t_{a} t_{b}$ be the induced metric on $\Sigma$ and let $D_{a}=h_{a}^{b} \nabla_{b}$, where $\nabla_{b}$ is the unique torsion-free derivative operator on $M$ compatible with $g_{a b}$. Thus $D_{a}$ is a derivative operator on $\Sigma$, but is not in general the covariant derivative compatible with $h_{a b}$.

Witten considers spinor fields $\alpha_{A}$ on $\Sigma$ which satisfy

$$
D_{A A^{\prime}} \alpha^{A}=0
$$

subject to the boundary condition that asymptotically for large $r$,

$$
\alpha^{A}={ }^{(0)} \alpha^{A}+O\left(r^{-1}\right),
$$

where ${ }^{(0)} \alpha^{A}$ is a constant spinor.

The existence of solutions to this equation with this boundary condition has recently been established [10-12].

Taking another derivative of (2.1), commuting derivatives, multiplying by $\bar{\alpha}_{A^{\prime}} t^{A A^{\prime}}$, and integrating over $\Sigma$, Witten obtains an expression which in the present notation is

$$
1 / 2 \int_{S}\left(D_{a} K_{b}\right) t^{b} d S^{a}=\int_{\Sigma}\left[-t^{A A^{\prime}}\left(D_{b} \alpha_{A}\right)\left(D^{b} \bar{\alpha}_{A^{\prime}}\right)+4 \pi T_{a b} t^{a} K^{b}\right] d \Sigma,
$$

where $S$ is the two-sphere at infinity on $\Sigma$ and $K_{a}=\alpha_{A} \bar{\alpha}_{A^{\prime}}$.

There are two remarkable features in this identity. First, commuting derivatives and integrating by parts both introduce the second fundamental form of $\Sigma$, but these terms all cancel in the final identity. Second, the curvature terms introduced by commuting derivatives are precisely the Einstein tensor $G_{a b}$ which can then be eliminated in favour of the stress tensor $T_{a b}$ by virtue of Einstein's equation. (In fact, Eq. (2.1) has also been considered by Sen and he too obtained the identity (2.3) [13].)

Now the right hand side of (2.3) is manifestly nonnegative: the second term because of the dominant energy condition, since $t^{a}$ and $K^{a}$ are future directed timelike and null respectively; and the first term because $h_{a b}$ is negative definite so that

1 After this paper was completed, we became aware of work by Israel and Nester [9]. They consider a different generalization of Witten's expression that does not involve the Weyl neutrino equation

2 We use standard spinor conventions: signature (+- - ) Riemann tensor defined by $2 \nabla_{[a} \nabla_{b]} \xi_{c}=-R_{a b c}^{m} \xi_{m}, R_{a b}=R_{a m b}^{m}$ 
by introducing an orthonormal frame on $\Sigma$ it can be expressed as the inner product between $t^{a}$ and the sum of three future directed null vectors.

Witten completes his proof of the positive energy conjecture at spatial infinity by showing that the left hand side of $(2.3)$ is related to $P_{a}^{\mathrm{ADM}}$ :

$$
1 / 2 \int_{S}\left(D_{a} K_{b}\right) t^{b} d S^{a}=4 \pi P_{a}^{\mathrm{ADM}(0)} K^{a},
$$

where ${ }^{(0)} K^{a}={ }^{(0)} \alpha^{A(0)} \alpha^{A^{\prime}}$ is the asymptotically constant null vector which is the limit of $K^{a}$.

Since ${ }^{(0)} \alpha^{A}$ was arbitrary, this shows that any null component of $P_{a}^{\mathrm{ADM}}$ is positive so that $P_{a}^{\mathrm{ADM}}$ must be future directed.

Equations (2.3) and (2.4) yield a relation between $P_{a}^{\mathrm{ADM}}$ and $T_{a b}$. This is a remarkable result and furnishes a simple proof of the positive energy conjecture at spatial infinity. However, it is restricted to a specific choice of three-surface $\Sigma$. Thus one reason for seeking a generalization of this relation is to place it in a four dimensional context which is more natural from the standpoint of general relativity. However, a more important reason for seeking a generalization is that it is not clear whether (2.3) can be used on an asymptotically null surface to relate $P_{a}^{\mathrm{B}}$ to $T_{a b}$. The issue is the apparently difficult question of whether the solutions to (2.1) exist with appropriate boundary conditions on an asymptotically null surface (see appendix).

We now show how (2.3) may be generalized to yield a relation between $P_{a}^{\text {ADM }}$ or $P_{a}^{\mathrm{B}}$ and $T_{a b}$. The cost of this generalization is that we lose the manifest positivity of the right hand side, but we shall return to this point in Sect. 4.

Let $\alpha^{A}$ be an arbitrary (smooth) spinor field on an asymptotically flat spacetime and, as before, let $K^{a}$ be the future directed null vector defined by $\alpha^{A}$ :

$$
K^{a}=\alpha^{A} \bar{\alpha}^{A^{\prime}} \text {. }
$$

Define a vector field $p^{a}$ by the following formula:

$$
\nabla^{b} \nabla_{[a} K_{b]}=4 \pi p_{a}
$$

i.e. $p_{a}$ is the "current for the Maxwell field with potential $K_{b}$." It is immediate from (2.6) that $p_{a}$ is divergence free, and that the conserved quantity obtained by integrating $p_{a}$ over a hypersurface $\Sigma$ may be expressed as an integral over an asymptotic two sphere $S$ :

$$
\int_{\Sigma} p_{a} d \Sigma^{a}=\frac{1}{4 \pi} \int_{S} \nabla_{[a} K_{b]} t^{b} d S^{a} .
$$

Of course, since $\alpha^{A}$ is arbitrary, (2.7) is a trivial identity and contains no information about the underlying spacetime. However, as we now show, if we impose certain conditions on the spinor field $\alpha^{A},(2.7)$ becomes a useful relation between local and total energy in general relativity.

The main condition we impose on the spinor field $\alpha^{A}$ is that it satisfies the Weyl neutrino equation

$$
\nabla_{A A^{\prime}} \alpha^{A}=0 .
$$


This implies that $K^{a}$ is divergence free and that $\nabla^{2} \alpha_{A}+\frac{1}{4} R \alpha_{A}=0$. Expanding the antisymmetrization in (2.6) and using these two facts yields

$$
p_{a}=-\frac{1}{4 \pi}\left(\nabla_{m} \alpha_{A}\right)\left(\nabla^{m} \bar{\alpha}_{A^{\prime}}\right)+T_{a b} K^{b}
$$

where we have used Einstein's equation to equate the curvature term which arises to the stress energy tensor $T_{a b}$ of the background space-time. This is in fact the only place that Einstein's equation is used in this analysis. (Note that $T_{a b}$ is not the stress energy tensor of the field $\alpha^{A}$. All the spin $1 / 2$ fields discussed here are test fields on the given background space-time.) Thus, for solutions to the Weyl equation, $p_{a}$ is directly related to the local stress energy of the space-time.

We now show that Witten's relation between local and total energy (2.3) is just a special case of (2.7). First recall the Cauchy initial value formulation for the Weyl equation [14]: Given a spinor field on a spacelike surface $\Sigma$, there exists a unique solution to the Weyl equation in the domain of dependence of $\Sigma, D(\Sigma)^{3}$, which agrees with the given field on $\Sigma$. In other words, the initial data is just the field itself and there are no constraint equations.

Now from the definition of $D_{a}$ on a surface $\Sigma$ with unit normal $t^{a}$ we have

$$
\nabla_{a}=D_{a}+t_{a} t^{b} \nabla_{b}
$$

For a solution $\alpha^{A}$ of the Weyl equation

$$
0=\nabla_{A A^{\prime}} \alpha^{A} \equiv D_{A A^{\prime}} \alpha^{A}+t_{A A^{\prime}} t^{b} \nabla_{b} \alpha^{A},
$$

so that

$$
t^{A A^{\prime}} \nabla_{b} \alpha_{A} \nabla^{b} \bar{\alpha}_{A^{\prime}}=t^{A A^{\prime}} D_{b} \alpha_{A} D^{b} \bar{\alpha}_{A^{\prime}}+2 t^{A A^{\prime}} D_{A^{\prime} B} \alpha^{B} D_{A B^{\prime}} \bar{\alpha}^{B^{\prime}}
$$

Using (2.9) and (2.10) we can rewrite Eq. (2.7) as follows:

$$
\begin{aligned}
\int_{\Sigma} p_{a} d \Sigma^{a}= & \int_{\Sigma}-\frac{1}{4 \pi}\left(t^{A A^{\prime}} D_{b} \alpha_{A} D^{b} \bar{\alpha}_{A^{\prime}}+2 t^{A A^{\prime}} D_{A^{\prime} B} \alpha^{B} D_{A B^{\prime}} \bar{\alpha}^{B^{\prime}}\right) \\
& +T_{a b} t^{a} K^{b} d \Sigma \\
& =-\frac{1}{4 \pi} \int_{S} 1 / 2\left(t^{a} \nabla_{a} K_{b}-t^{a} D_{b} K_{a}\right) d S^{b}
\end{aligned}
$$

Thus if we choose initial data for the Weyl equation such that $D_{A A^{\prime}} \alpha^{A}=0$ and $\alpha^{A} \rightarrow^{(0)} \alpha^{A}$ asymptotically on $\Sigma$, then (2.11) reduces to precisely Witten's relation (2.3). In particular, the boundary integral is equal to (a null component of) $P_{a}^{\text {ADM }}$. By evolving this initial data we obtain a conserved vector field $p_{a}$ which has the property that its integral over any asymptotically flat spacelike surface yields the total ADM energy-momentum of the spacetime. (Actually, all that has been shown so far is that the integral yields $P_{a}^{\text {ADM }}$ for any surface which agrees with $\Sigma$ asymptotically. Although we haven't performed a detailed analysis at spatial

3 Recall that $D(\Sigma)$ is the set of all points $p$ to the future (past) of $\Sigma$ such that every past (future) directed timelike curve from $p$ without endpoint intersects $\Sigma$ 
infinity, we expect that since $p_{a}$ falls off like $r^{-4}$ at large spacelike distances, its integral over any asymptotically flat surface - even those boosted with respect to $\Sigma$ - will again yield $P_{a}^{\mathrm{ADM}}$.)

The vector field $p_{a}$ can therefore be interpreted as an energy-momentum density for gravity. However one must keep in mind that $p_{a}$ is not uniquely determined by the spacetime: First, one has the freedom of choosing the constant spinor ${ }^{(0)} \alpha^{A}$ that the initial data approaches. This choice determines which null component of $P_{a}^{\mathrm{ADM}}$ is being measured. Second, one has the freedom of choosing the initial surface $\Sigma$. Different choices of $\Sigma$ will yield different vector fields $p_{a}$. Finally, one has the freedom of constructing $p_{a}$ from solutions $\alpha^{A}$ to the Weyl equation which do not satisfy (2.1) on any spacelike surface. One need only require that near spatial infinity $\alpha^{A}={ }^{(0)} \alpha^{A}+O\left(r^{-1}\right)$ to ensure that the boundary integral yields the ADM energy momentum. This follows from Nester's reformulation [15] of Witten's argument ${ }^{4}$. Thus there is a large collection of conserved vectors $p_{a}$ with the property that their integrals are related to $P_{a}^{\mathrm{ADM}}$. This nonuniqueness seems to be the reflection in the present framework of the well-known fact that there is no unique energy density of the gravitational field.

We have now achieved half of our initial objective Namely, we have a (four dimensional) relation between local energy and total ADM energy at spatial infinity. The second half of our objective is to obtain a similar relation between local energy and total Bondi energy at null infinity.

In one respect the analysis at null infinity is slightly more complicated than that at spatial infinity. This concerns the definition of an "asymptotically constant spinor." Perhaps the most natural definition is to call a spinor field asymptotically constant if its covariant derivative tends to zero. Near spatial infinity, the Riemann tensor falls off as $O\left(r^{-3}\right)$, so spinors which are asymptotically constant in this sense do exist. Near null infinity, however, in the presence of gravitational radiation, the Riemann tensor falls off only as $O\left(r^{-1}\right)$. Bramson [16] has shown that under these conditions, $\nabla_{m} \alpha_{A} \rightarrow 0$ near null infinity if and only if $\alpha^{A} \rightarrow 0$ as well. He has thus proposed another definition for when a spinor field is "asymptotically constant." Essentially, he requires that the symmetrized derivative of $\alpha_{A}$ rather than the full covariant derivative should vanish at null infinity. Bramson has shown that there always exist spinors satisfying this condition. In Sect. 3, we shall discuss this condition in terms of the conformally rescaled space-time and show that there do exist solutions to the Weyl equation with this asymptotic behaviour. In the remainder of this section, we stay in the physical space-time and show that such solutions provide us with our desired relation involving the Bondi energy.

4 Nester [15] shows that if $\alpha^{A}={ }^{(0)} \alpha^{A}+O\left(r^{-1}\right)$, then

$$
\frac{1}{8 \pi} \int_{S} F_{a b} t^{b} d S^{a}={ }^{(0)} K^{a} P_{a}^{\mathrm{ADM}},
$$

where

$$
F_{a b}=\left[\bar{\alpha}^{M^{\prime}} \nabla_{M^{\prime}(A} \alpha_{B)}-\alpha_{(A} \nabla_{B) M^{\prime}} \bar{\alpha}^{M^{\prime}}\right] \varepsilon_{A^{\prime} B^{\prime}}+\text { c.c. . }
$$

If $\alpha_{A}$ is a solution to the Weyl equation, then $F_{a b}=2 \nabla_{[a} K_{b]}$ and Nester's boundary integral is equivalent to the right hand side of $(2.7)$ 
Let $M, g_{a b}$ be asymptotically flat at null infinity, i.e., there exists (Bondi) coordinates $(u, r, \theta, \varphi)$ defined outside a world tube such that the metric takes the form:

$$
\begin{aligned}
d s^{2}= & 2 d u d r-r^{2}\left(d \theta^{2}+\sin ^{2} \theta d \varphi^{2}\right) \\
& +O\left(r^{-1}\right) d r \times(\text { differentials not involving } d r) \\
& +O(r) \times(\text { differentials not involving } d r),
\end{aligned}
$$

where the $u=$ const surfaces are null, and $r$ is an affine parameter on each null geodesic generator. It is convenient to adopt the NP formalism [17]. We introduce a null tetrad as follows. Let $l_{a}=\nabla_{a} u$. Let $n^{a}$ be the ingoing null vector orthogonal to the spheres of constant $u$ and $r$ normalised by $l_{a} n^{a}=1$. Finally we choose complex conjugate null vectors $m^{a}, \bar{m}^{a}$ normalised by $m^{a} \bar{m}_{a}=-1$ and tangent to the spheres of constant $u$ and $r$. Note that $n^{a}$ is not propagated parallel along $l^{a}$. This tetrad defines a spinor dyad $\left(o^{A}, l^{A}\right)$ with $l^{a}=o^{A} \bar{o}^{A^{\prime}}, n^{a}=l^{A} \bar{l}^{A^{\prime}}, m^{a}=o^{A} \bar{l}^{A^{\prime}}, o_{A} l^{A}=1$ and $l^{m} \nabla_{m} o^{A}=0$.

Recall that the spin coefficients are defined as follows:

$$
\begin{aligned}
& \kappa=o^{A} D o_{A} \\
& \tau=o^{A} \Delta o_{A} \\
& \sigma=o^{A} \delta o_{A} \\
& \varrho=o^{A} \bar{\delta} o_{A} \\
& \pi=l^{A} D l_{A} \\
& \nu=l^{A} \Delta l_{A} \\
& \mu=l^{A} \delta l_{A} \\
& \lambda=l^{A} \bar{\delta} l_{A} \\
& \varepsilon=l^{A} D o_{A} \\
& \gamma=l^{A} \Delta o_{A} \\
& \beta=l^{A} \delta o_{A} \\
& \alpha=l^{A} \bar{\delta} o_{A},
\end{aligned}
$$

where $D=l^{a} \nabla_{a}, \Delta=n^{a} \nabla_{a}, \delta=m^{a} \nabla_{a}$, and $\bar{\delta}=\bar{m}^{a} \nabla_{a}$. For the above tetrad, $\kappa=\varepsilon=0$, $\tau=\bar{\pi}=\bar{\alpha}+\beta, \varrho=\bar{\varrho}$, and $\mu=\bar{\mu}[18]$. We will use one component of the Weyl spinor:

$$
\psi_{2}=\psi_{A B C D} O^{A} O^{B} l^{C} l^{D} .
$$

The Bondi energy momentum is defined in terms of the asymptotic values of $r^{2} \sigma$ and $r^{3} \psi_{2}$, denoted $\sigma^{0}$ and $\psi_{2}^{0}$. For an asymptotic translation $V^{a}$ :

$$
V^{a} P_{a}^{B} \equiv-\frac{1}{4 \pi} \int_{S}\left(V^{a} l_{a}\right) \frac{\left(\psi_{2}^{0}+\sigma^{0} \dot{\bar{\sigma}}^{0}\right)}{r^{2}} d S,
$$

where $S$ is an asymptotic two-sphere, and $a$ dot denotes $d / d u$.

The boundary condition on $\alpha^{A}$ can be expressed in terms of its components in the spinor basis:

$$
\alpha^{A}=X o^{A}+Y l^{A} .
$$


Bramson's condition for $\alpha^{A}$ to be asymptotically constant is that, for large $r$,

$$
\begin{gathered}
X=X_{0}(\theta, \phi)+X_{1}(u, r, \theta, \varphi), \\
Y=Y_{0}(\theta, \phi)+Y_{1}(u, r, \theta, \phi),
\end{gathered}
$$

where $X_{0}$ and $Y_{0}$ are linear combinations of $l=1 / 2$ spin-weighted spherical harmonics [19]:

$$
\begin{gathered}
X_{0}=a_{-1 / 2} Y_{1 / 21 / 2}+b_{-1 / 2} Y_{1 / 2-1 / 2}, \\
Y_{0}=-a_{1 / 2} Y_{1 / 21 / 2}-b_{1 / 2} Y_{1 / 2-1 / 2}
\end{gathered}
$$

for some complex constants $a$ and $b$; and $X_{1} \rightarrow 0$ as $r^{-1}$ and $Y_{1} \rightarrow 0$ faster than $r^{-1}$.

We can now prove the following:

Proposition 1. Let $\alpha^{A}$ be a solution to the Weyl equation on a space-time $M, g_{a b}$ which is asymptotically flat at null infinity. If $\alpha^{A}$ satisfies (2.14) and (2.15) then

$$
\int_{\Sigma^{\prime}} p_{a} d \Sigma^{a}=P_{a}^{B(0)} K^{a}
$$

for all asymptotically null $\Sigma^{\prime}$, where ${ }^{(0)} K^{a}$ is the null vector defined by ${ }^{(0)} \alpha^{A}=X_{0} o^{A}+Y_{0} l^{A}$.

Proof. Fix an asymptotically null surface $\Sigma^{\prime}$. Choose a system of Bondi coordinates such that $\Sigma^{\prime}$ asymptotically approaches a $u=$ const null cone [3]. Define a null basis $l^{a}, n^{a}, m^{a}, \bar{m}^{a}$ in terms of these coordinates as described above. Then the twosurface bivector in (2.7) is $l^{[a} n^{b]}$ so that (2.7) becomes

$$
\int_{\Sigma^{\prime}} p_{a} d \Sigma^{a}=\frac{1}{8 \pi} \int_{S}\left(n_{a} D K^{a}-l_{a} \Delta K^{a}\right) d S .
$$

The Weyl equation may be written

$$
\begin{aligned}
& l^{A} D \alpha_{A}-o^{A} \bar{\delta} \alpha_{A}=0, \\
& l^{A} \delta \alpha_{A}-o^{A} \Delta \alpha_{A}=0 .
\end{aligned}
$$

Using (2.17) and (2.13), the boundary term in (2.16) becomes

$$
\frac{1}{8 \pi} \int_{S}\left(\bar{Y} l^{A} \delta \alpha_{A}+\bar{X} o^{A} \bar{\delta} \alpha_{A}+\text { complex conjugate }\right) d S
$$

which, using the definition of the spin coefficients and (2.13) again, is

$$
\int_{\Sigma^{\prime}} p_{a} d \Sigma^{\prime a}=\frac{1}{4 \pi} \int_{S}(X \bar{X} \varrho+Y \bar{Y} \mu+2 \operatorname{Re}(\bar{Y} \delta X+\beta \bar{Y} X)) d S .
$$

Here we have used the fact that $\varrho$ and $\mu$ are real for our tetrad. The asymptotic values of the spin coefficients appearing in (2.18) have been found to sufficiently high order by Exton et al. [20], in a basis in which $n^{a}$ is propagated parallel along $l^{a}$. To use their results we must perform a null rotation

$$
l^{A} \rightarrow l^{A}-\bar{\omega} O^{A}
$$


with $\bar{\omega}$ as in [20]. We then find

$$
\begin{aligned}
\varrho & =-\frac{1}{r}+O\left(r^{-3}\right), \\
\mu & =-\frac{1}{r}-\frac{1}{r^{2}}\left(\psi_{2}^{0}+\sigma^{0} \dot{\bar{\sigma}}^{0}+\circlearrowright^{2} \bar{\sigma}^{0}\right)+O\left(r^{-3}\right), \\
(\delta+\beta) X & =\left(-\frac{1}{r} \circlearrowright+\frac{\sigma^{0}}{r^{2}} \bar{\delta}+O\left(r^{-3}\right)\right) X,
\end{aligned}
$$

where $\partial$ is the standard differential operator on the sphere [19]. Using these expressions in (2.18), we see that the $O\left(r^{-1}\right)$ term in the boundary integral vanishes by virtue of (2.14) and (2.15), and the $O\left(r^{-2}\right)$ term yields

$$
\begin{aligned}
\int_{\Sigma^{\prime}} p_{a} d \Sigma^{a} & =-\frac{1}{4 \pi} \int_{S} \frac{1}{r^{2}} Y_{0} \bar{Y}_{0}\left(\psi_{2}^{0}+\sigma^{0} \dot{\bar{\sigma}}^{0}+\delta^{2} \bar{\sigma}^{0}\right) d S \\
& =P_{a}^{B(0)} K^{a},
\end{aligned}
$$

since the last term integrates to zero. Since $\Sigma^{\prime}$ was arbitrary, this completes the proof.

Since $p_{a}$ is conserved and its integral over any asymptotically null slice yields the Bondi energy at that time, its integral over a null surface spanning two asymptotic two-surfaces at different retarded times must reproduce the BondiSachs mass loss formula $[2,3]$. We can verify this directly by computing the flux $n^{a} p_{a}$. Suppose first that $T_{a b}$ vanishes in a neighbourhood of null infinity. Then by (2.9),

$$
n^{a} p_{a}=-\frac{1}{4 \pi} l^{A} \bar{\imath}^{A^{\prime}}\left(D \alpha_{A} \Delta \bar{\alpha}_{A^{\prime}}+\Delta \alpha_{A} D \bar{\alpha}_{A^{\prime}}-\delta \alpha_{A} \bar{\delta} \bar{\alpha}_{A^{\prime}}-\bar{\delta} \alpha_{A} \delta \bar{\alpha}_{A^{\prime}}\right) .
$$

Since $\alpha_{A}$ is asymptotically constant, the first three terms on the right fall off faster than $r^{-2}$. For the last term, we have

$$
{ }^{A} \bar{\delta} \alpha_{A}=\bar{\delta} X+\alpha X+\lambda Y=\frac{1}{r} \dot{\bar{\sigma}}^{0} Y_{0}+O\left(r^{-2}\right),
$$

where we have used the asymptotic expansions of $\alpha, \lambda[20]$ and the fact that $\bar{\delta} X_{0}=0$. Therefore,

$$
n^{a} p_{a}=\frac{1}{4 \pi r^{2}} Y_{0} \bar{Y}_{0} \dot{\sigma}^{0} \dot{\bar{\sigma}}^{0}+O\left(r^{-3}\right),
$$

which gives precisely the Bondi-Sachs flux for $P_{a}^{\mathrm{B}(0)} K^{a}$. Notice that the leading term on the right hand side of (2.21) is non-negative, i.e. gravitational waves always carry away positive energy from the system. If $T_{a b}$ does not vanish in a neighborhood of null infinity, then there is the additional term $T_{a b} n^{a} K^{b}$ on the right hand side of (2.21), which is just the contribution of the matter fields to the energy radiated away. 


\section{Existence of Solutions}

We begin this section with a discussion of Bramson's conditions for asymptotically constant spinors at null infinity, and translate some of the results of the previous section into the conformally rescaled spacetime. We then show that solutions to the Weyl equation with Bramson's boundary conditions do, indeed, exist.

We first review the definition of asymptotic flatness at null infinity in terms of a conformal completion of the spacetime [21]:

Definition. A spacetime $M, g_{a b}$ is asymptotically flat at null infinity if there exists a manifold with boundary, $\hat{M}=M \cup \mathscr{I}$, consisting of $M$ with boundary $\mathscr{I}$ attached together with a smooth Lorentz metric $\hat{g}_{a b}$ and smooth scalar field $\Omega$ on $\hat{M}$ such that:

1) on $M, \hat{g}_{a b}=\Omega^{2} g_{a b}$

2) at $\mathscr{I}, \Omega=0, \hat{n}_{a}=\hat{V}_{a} \Omega \neq 0, \hat{n}_{a} \hat{n}^{a}=0$, and $\hat{\nabla}_{a} \hat{n}_{b}=0$,

3) $\mathscr{I}$ consists of two pieces $\mathscr{I}^{+}$and $\mathscr{I}^{-}$, each $S^{2} \times \mathbb{R}$ with $\mathbb{R}$ being the null generators and on each of which $\hat{n}_{a}$ is complete.

This definition insures that asymptotically $\left(M, g_{a b}\right)$ resembles Minkowski spacetime both locally (Conditions 1 and 2) and globally (Condition 3). In Bondi coordinates, we can choose the conformal factor to be $\Omega=r^{-1}$ near null infinity.

To discuss the behaviour of a spinor field $\alpha^{A}$ near $\mathscr{I}$, we must decide how to scale $\alpha^{A}$ under conformal transformations. The Weyl neutrino equation is conformally invariant provided one scales $\alpha^{A}$ according to

$$
\hat{\alpha}^{A}=\Omega^{-2} \alpha^{A},
$$

since in this case

$$
\hat{\nabla}_{A A^{\prime}} \hat{\alpha}^{A}=\Omega^{-2} \nabla_{A A^{\prime}} \alpha^{A}
$$

Although this scaling has the advantage that $\hat{\alpha}^{A}$ satisfies a simple equation in the unphysical spacetime, it also has a great disadvantage: If $\alpha^{A}$ is asymptotically constant in the physical spacetime, then $\hat{\alpha}^{A}$ defined by (3.1) is singular on $\mathscr{I}$. Since we wish to clarify the asymptotic boundary conditions on $\alpha^{A}$, we want a field which is regular on $\mathscr{I}$. We therefore adopt the following scaling for $\alpha^{A}$ :

$$
\hat{\alpha}^{A}=\alpha^{A} \text {. }
$$

With this scaling, the twistor operator is conformally invariant:

$$
\hat{\nabla}^{A^{\prime}}{ }_{(A} \hat{\alpha}_{B)}=\nabla^{A^{\prime}}{ }_{(A} \alpha_{B)} \text {. }
$$

We can now state Bramson's boundary conditions at null infinity:

Definition. A smooth solution $\alpha^{A}$ to the Weyl equation is asymptotically constant at null infinity if

1) $\hat{\alpha}^{A}=\alpha^{A}$ has a smooth, non-vanishing limit on $\mathscr{I}$,

2) $\hat{\nabla}_{A^{\prime}\left(A^{\prime}\right)} \hat{\alpha}_{B)}=0$ on $\mathscr{I}$.

Roughly speaking, the first condition says that $\alpha^{A}$ has some limit along each null direction, and the second condition says that these limits are in fact all the same. Bramson [16] has shown that if $\hat{\alpha}^{A}$ is asymptotically constant in the above 
sense, then $\hat{\alpha}^{A} \hat{\bar{\alpha}}^{A^{\prime}}$ evaluated on $\mathscr{I}$ is a multiple of $\hat{n}^{a}$ and in fact defines a null translation ${ }^{(0)} K^{a}$ in the BMS (Bondi-Metzner-Sachs) group (the asymptotic symmetry group of null infinity [22]).

One can now see that the asymptotic boundary integral for $p^{a}(2.7)$ is just a special case of the Geroch-Winicour formulation of asymptotic linkages [23]. Since $K^{a}$ is divergence free in the physical spacetime, $\hat{K}^{a}$ satisfies the GerochWinicour gauge condition for extensions of BMS generators, and the boundary integral (2.7) is precisely the asymptotic linkage in this gauge. This provides another way of showing that for asymptotically constant solutions, the integral of $p^{a}$ is related to the Bondi four-momentum.

Although one can obtain null translations (and by linear combinations, all translations) from solutions to the Weyl equation, in the presence of gravitational radiation one cannot obtain other BMS generators. We will show at the end of this section that if $\ddot{\sigma}^{0} \neq 0$ at some retarded time, and $\alpha^{A}$ satisfies $\nabla_{A A^{\prime}} \alpha^{A}=0$ and admits a smooth extension to $\mathscr{I}^{+}$, then $\alpha^{A}$ is necessarily asymptotically constant.

Since $p_{a}$ is conserved in the physical spacetime,

$$
\hat{p}_{a}=\Omega^{-2} p_{a}
$$

is conserved in the unphysical spacetime, and yields the same conserved quantity when integrated over a spacelike surface. We now show that if $M, g_{a b}$ is asymptotically flat and $\hat{T}_{a b}=\Omega^{-2} T_{a b}$ has a smooth extension to $\mathscr{I},{ }^{5}$ then $\hat{p}_{a}$ has a smooth extension to $\mathscr{I}$ as well. Using the fact that $\alpha^{A}$ is a smooth solution to the Weyl equation in the physical spacetime, we can rewrite (2.9) in the form:

$$
p_{a}=-\frac{1}{4 \pi} g^{m n} \nabla_{M^{\prime}\left(M^{\prime}\right.} \alpha_{A)} \nabla_{N\left(N^{\prime}\right.} \bar{\alpha}_{\left.A^{\prime}\right)}+T_{a b} K^{b} .
$$

In this form it is clear that under (3.3), $p_{a}$ is conformally invariant. Replacing the fields on the right hand side by the conformally scaled fields and using (3.4) yields

$$
p_{a}=-\frac{1}{4 \pi} \hat{g}^{m n} \hat{\nabla}_{M^{\prime}\left(M^{\prime}\right.} \hat{\alpha}_{A)} \hat{V}_{N\left(N^{\prime}\right.} \hat{\bar{\alpha}}_{\left.A^{\prime}\right)}+\Omega^{2} \hat{T}_{a b} \hat{K}^{b} .
$$

Since $\hat{\nabla}_{M^{\prime}\left(M^{\prime}\right.} \hat{\alpha}_{A)}$ vanishes on $\mathscr{I}$,

$$
\hat{S}_{M^{\prime} M A}=\Omega^{-1} \hat{V}_{M^{\prime}\left(M^{\prime}\right.} \hat{\alpha}_{A)}
$$

is smooth on $\mathscr{I}$. Therefore

$$
\hat{p}_{a}=-\frac{1}{4 \pi} \hat{S}_{M^{\prime} M A} \hat{\bar{S}}^{M M^{\prime}}{ }_{A^{\prime}}+\hat{T}_{a b} \hat{K}^{b}
$$

is smooth on $\mathscr{I}$. In particular, $\hat{p}_{a} \hat{n}^{a}$ is well defined on $\mathscr{I}$ and yields precisely the flux of the ${ }^{(0)} K^{a}=\left.\hat{\alpha}^{A} \hat{\bar{\alpha}}^{A^{\prime}}\right|_{\mathscr{I}}$ component of the Bondi energy-momentum. By taking linear combinations of different $p_{a}$ 's one can obtain the flux of any component of $P_{a}^{\mathrm{B}}$.

We now turn to the question of whether there exist solutions to the Weyl equation which are asymptotically constant in the above sense.

5 This is a condition on the asymptotic behaviour of the physical stress energy which admits a large class of physically interesting examples 
Proposition 2. Let $M, g_{a b}$ be a spacetime (with spin structure) which is asymptotically flat at null infinity. Let $\Sigma^{\prime}$ be an asymptotically null surface in $M$. Then there exist solutions to the Weyl equation in the domain of dependence of $\Sigma^{\prime}, D\left(\Sigma^{\prime}\right)$, which are asymptotically constant.

Outline of Proof. The idea of the proof is to choose a spinor ${ }^{(0)} \alpha^{A}$ which satisfies the boundary conditions, and then solve $\nabla_{A A^{\prime}}{ }^{(1)} \alpha^{A}=-\nabla_{A A^{\prime}}{ }^{(0)} \alpha^{A}$ for a spinor ${ }^{(1)} \alpha^{A}$ that has the appropriate fall-off behaviour so that $\alpha^{A}={ }^{(0)} \alpha^{A}+{ }^{(1)} \alpha^{A}$ is an asymptotically constant solution to the Weyl equation.

We introduce a Bondi coordinate system in a neighbourhood of $\mathscr{I}^{+}$such that $\Sigma^{\prime}$ intersects $\mathscr{I}^{+}$in a $u=$ const cross-section. Let $O^{A}, l^{A}$ be a spinor basis adapted to these coordinates, as described earlier. We first re-express the boundary conditions and the asymptotic form of the Weyl equation in terms of the components $X$ and $Y$ of a spinor $\alpha^{A}=X O^{A}+Y l^{A}$.

The first boundary condition (together with the fact that $\alpha^{A}$ is a solution to the Weyl equation) is equivalent to the statement that for large $r$

$$
\begin{gathered}
X \rightarrow X_{0}(u, \theta, \phi), \\
Y \rightarrow Y_{0}(u, \theta, \phi) .
\end{gathered}
$$

The second condition, as Bramson has shown (Eq. (4.11) of [16]) is equivalent to

$$
\begin{aligned}
\bar{ð} Y_{0}-X_{0} & =0, \\
\dot{Y}_{0} & =0, \\
ð Y_{0} & =0,
\end{aligned}
$$

and

$$
\lim _{r \rightarrow \infty} r\left(Y-Y_{0}\right)=0
$$

where ${ }^{\cdot}=\partial / \partial u$. Of course (3.11a) and (3.11b) imply that $\dot{X}_{0}=0$ as well. The general solution to $(3.11 \mathrm{a}-\mathrm{c})$ is given by $(2.15)$. (This is why that equation was imposed in the previous section.)

The Weyl equation in the NP formalism (2.17) with $\alpha^{A}$ expanded in terms of $X$ and $Y$ becomes

$$
\begin{aligned}
D X-\varrho X & =-\bar{\delta} Y+\alpha Y-\pi Y, \\
\delta X+\beta X-\tau X & =-\Delta Y+\gamma Y-\mu Y,
\end{aligned}
$$

where we have used the fact that the spin coefficient $\varepsilon$ vanishes for this tetrad. Applying the necessary null rotation to the formulae of [20] we find that $(3.12 a, b)$ become

$$
\begin{aligned}
\frac{\partial X}{\partial r}+\frac{1}{r} X+O\left(r^{-3}\right) X & =\frac{1}{r} \bar{\delta} Y-\frac{1}{r^{2}} \bar{\sigma}^{0} \partial Y+O\left(r^{-3}\right) Y \\
-\frac{1}{r} \partial X+O\left(r^{-2}\right) X & =-\dot{Y}+\frac{\partial Y}{\partial r}+\frac{1}{r} Y+O\left(r^{-2}\right) Y .
\end{aligned}
$$


Let $X_{0}$ and $Y_{0}$ be defined by (2.15) and let

$$
{ }^{(0)} \alpha^{A}=\left(X_{0} o^{A}+Y_{0} l^{A}\right) f(r),
$$

where $f$ is a smooth function of $r$ satisfying $f(r)=1$ for $r>R_{1}$ and $f(r)=0$ for $r<R_{2}$, where $R_{1}>R_{2}$ and both $R_{\imath}$ lie in the domain of the Bondi coordinates. The spinor ${ }^{(0)} \alpha^{A}$ clearly satisfies the boundary conditions (3.10) and (3.11) required for asymptotic constancy. Since ${ }^{(0)} \alpha^{A}$ is independent of $r$ (for $r>R_{\imath}$ ), the behavior of $\nabla_{A A^{\prime}}{ }^{(0)} \alpha^{A}$ for large $r$ is the same as that of the spin coefficients in (3.12). These spin coefficients all admit expansions in powers of $r^{-1}$ [20]. Substituting ${ }^{(0)} \alpha^{A}$ into (3.13) one finds that the power series expansion of $\nabla_{A A^{\prime}}{ }^{(0)} \alpha^{A}$ begins at order $r^{-2}$ for the coefficient of $o_{A}$ and order $r^{-3}$ for the coefficient of $l_{A}$ :

$$
\nabla_{A A^{\prime}}{ }^{(0)} \alpha^{A}=P\left(r^{-2}\right) o_{A}+P\left(r^{-3}\right) l_{A}
$$

where $P\left(r^{-n}\right)$ denotes a power series in $r^{-1}$ beginning with a term of order $r^{-n}$.

We now pass to the unphysical spacetime. Since $o_{A}$ is parallel propagated along $l^{a}$ in the physical spacetime, $\hat{o}_{A}=O_{A}$ is parallel propagated in the unphysical spacetime, and hence is smooth (and non-vanishing) on $\mathscr{I}^{+}$. Since $\hat{g}_{a b}=\Omega^{2} g_{a b}$ is smooth on the boundary, $\hat{\varepsilon}_{A B}=\Omega \varepsilon_{A B}$ is smooth and hence $\hat{l}_{A}=\Omega l_{A}$ will be smooth on $\mathscr{I}^{+}$as well. Therefore

$$
\hat{o}_{A}=o_{A}, \hat{l}_{A}=\Omega l_{A}
$$

defines a smooth spinor basis in a neighbourhood of null infinity.

From Eqs. (3.15), (3.16) and the fact that $\Omega=r^{-1}$ near $\mathscr{I}^{+}$, one can show that

$$
\hat{\varrho}_{A^{\prime}} \equiv-\Omega^{-2} \nabla_{A A^{\prime}}{ }^{(0)} \alpha^{A}
$$

admits a smooth extension to $\mathscr{I}^{+}$. Choose any spinor field on $\Sigma^{\prime}$ with compact support. Let ${ }^{(1)} \hat{\alpha}^{A}$ be the solution in $\overline{D\left(\Sigma^{\prime}\right)}$ (where the closure is taken in $\hat{M}$ ) to the equation

$$
\hat{\nabla}_{A A^{\prime}}{ }^{(1)} \hat{\alpha}^{A}=\hat{\varrho}_{A^{\prime}}
$$

with this initial data. Since (3.18) is a hyperbolic equation with smooth coefficients and smooth source, it admits [14] solutions ${ }^{(1)} \alpha^{A}$ which are smooth on $\mathscr{I}^{+}$. [The fact that $\mathscr{I}^{+}$is a boundary to the spacetime is irrevelant here since one can always extend the spacetime and $\hat{\varrho}_{A^{\prime}}$ beyond $\mathscr{I}^{+}$and solve (3.18) in the extended spacetime.]

Returning to the physical spacetime, let

$$
{ }^{(1)} \alpha^{A}=\Omega^{2(1)} \hat{\alpha}^{A} \text {. }
$$

Re-expressing ${ }^{(1)} \alpha^{A}$ in terms of the physical spinor basis yields

$$
{ }^{(1)} \alpha^{A}=O\left(r^{-1}\right) O^{A}+O\left(r^{-2}\right) l^{A} .
$$

Now define $\alpha^{A}={ }^{(0)} \alpha^{A}+{ }^{(1)} \alpha^{A}$. From (3.14) and (3.20) $\alpha^{A}$ is asymptotically constant. Furthermore

$$
\nabla_{A A^{\prime}} \alpha^{A}=\nabla_{A A^{\prime}}{ }^{(0)} \alpha^{A}+\nabla_{A A^{\prime}}{ }^{(1)} \alpha^{A}
$$


But since the Weyl equation is conformally invariant under (3.19)

$$
\nabla_{A A^{\prime}}{ }^{(1)} \alpha^{A}=\Omega^{2} \hat{\nabla}_{A A^{\prime}}{ }^{(1)} \hat{\alpha}^{A}=\Omega^{2} \hat{\varrho}_{A^{\prime}}=-\nabla_{A A^{\prime}}{ }^{(0)} \alpha^{A} .
$$

Therefore $\nabla_{A A^{\prime}} \alpha^{A}=0$. This completes the proof.

We conclude this section by proving a partial converse to Proposition 2. That is, in the presence of gravitational radiation, $\ddot{\sigma}^{0} \neq 0$, all solutions to the Weyl equation which admit a smooth extension to null infinity are asymptotically constant.

Proposition 3. Let $M, g_{a b}$ be a spacetime which is asymptotically flat at null infinity and has $\ddot{\sigma}^{0} \neq 0$ on at least one cross-section $C$ of $\mathscr{I}^{+}$. Let $\alpha^{A}$ be a smooth solution to the Weyl equation in a neighbourhood of $\mathscr{I}^{+}$such that $\hat{\alpha}^{A}=\alpha^{A}$ admits a smooth extension to $\mathscr{I}^{+}$. Then $\hat{\nabla}_{A^{\prime}(A} \hat{\alpha}_{B)}=0$ on $\mathscr{I}^{+}$.

Proof. We introduce Bondi coordinates in which the cross-section $C$ is given by $u=0$. Since $\hat{\alpha}^{A}$ is smooth on $\mathscr{I}^{+}$, it can be expanded in a power series (plus remainder) in $\Omega$. Using the transformation of the spinor basis (3.16) this implies that $\alpha^{A}$ can be expanded in the following power series in $\Omega=1 / r$ :

$$
\alpha^{A}=X o^{A}+Y l^{A}
$$

with

$$
\begin{aligned}
& X=X_{1} r+X_{0}+X_{-1} r^{-1}+O\left(r^{-2}\right), \\
& Y=Y_{0}+Y_{-1} r^{-1}+O\left(r^{-2}\right) .
\end{aligned}
$$

Since $\alpha^{A}$ is a solution to the Weyl equation, it must satisfy $(3.13 \mathrm{a}, \mathrm{b})$. Taking the limit of these equations as $r \rightarrow \infty$ and using the above expansions yields

$$
\begin{aligned}
& (3.13 \mathrm{a}) \Rightarrow\left\{\begin{array}{l}
X_{1}=0, \\
\bar{\partial} Y_{0}-X_{0}=0, \\
\bar{\partial} Y_{-1}-\bar{\sigma}^{0} ð Y_{0}=0,
\end{array}\right. \\
& (3.13 b) \Rightarrow\left\{\begin{array}{l}
\dot{Y}_{0}=0, \\
ð X_{0}+Y_{0}-\dot{Y}_{-1}=0 .
\end{array}\right.
\end{aligned}
$$

Equation (3.23a) shows that the first condition in the definition of asymptotic constancy is indeed equivalent to (3.10). Equations (3.23b) and (3.24a) are precisely $(3.11 \mathrm{a}, \mathrm{b})$. These two equations imply $\dot{X}_{0}=0$. Taking the $u$ derivative of $(3.24 \mathrm{~b})$ yields $\ddot{Y}_{-1}=0$. Taking two $u$ derivatives of (3.23c) now yields $\ddot{\bar{\sigma}}^{0} \not \partial Y_{0}=0$. Since $\ddot{\bar{\sigma}}^{0} \neq 0$ when $u=0$, we conclude that for $u=0$

$$
ð Y_{0}=0 .
$$

But since $\dot{Y}_{0}=0,(3.25)$ holds for all $u$.

Substituting (3.25) back into (3.23c) yields $\bar{\delta} Y_{-1}=0$ which implies that

$$
Y_{-1}=0
$$

(since $Y_{-1}$ has spin weight $\left.+1 / 2\right)$. But (3.25) and (3.26) are precisely (3.11c, d). Since $(3.11 \mathrm{a}-\mathrm{d})$ are satisfied, $\hat{\nabla}_{A^{\prime}\left(A^{\prime}\right.} \hat{\alpha}_{B)}=0$ on $\mathscr{I}^{+}$. 
By a slight modification of this proof one can show that the condition of the existence of a cross-section with gravitational radiation $(\ddot{\sigma} \neq 0)$ can be replaced by the existence of a cross-section with no news $\left(\dot{\sigma}^{0}=0\right)$, and the result will still hold. As mentioned earlier, Proposition 3 shows that there do not exist solutions $\alpha^{A}$ of the Weyl equation such that $\hat{\alpha}^{A}=\alpha^{A}$ approach asymptotic symmetries (BMS generators) other than translations.

\section{Conclusion}

In this paper we have considered asymptotically constant solutions $\alpha^{A}$ to the Weyl neutrino equation on an asymptotically flat spacetime. We have shown that associated with these solutions there exist a collection of conserved vector fields $p_{a}$. These vector fields depend explicitly on the local stress energy of the matter and have the property that their integrals over spacelike surfaces yield the total ADM or Bondi four-momentum of the spacetime.

This relation between local and total energy in general relativity becomes more useful when one has a statement about the sign of the energy integral. That is, a statement about when $p_{a}$ is a future directed timelike or null vector. Witten has given a specific prescription for choosing $p_{a}$ to be future directed on an asymptotically flat surface $\Sigma$. Namely, choose asymptotically constant initial data for the Weyl equation which satisfies (2.1) on $\Sigma$. However, it is clear that this is not the only choice of initial data for which $p_{a}$ will be future directed. Thus we have a generalization of Witten's proof of the positivity of the ADM energy. Recently, it has been shown [24] that this generalization can be used to prove the positivity of the Bondi energy. In other words, one can choose asymptotically constant initial data (not satisfying 2.1) on an asymptotically null surface $\Sigma^{\prime}$ such that $p_{a}$ is future directed on $\Sigma^{\prime}$.

In order to interpret $p_{a}$ as an "energy-momentum density" for a gravitating system, one would like $p_{a}$ to be future directed everywhere in the spacetime - not just on one spacelike surface. We are thus led to the following conjecture:

Conjecture. Let $M, g_{a b}$ be asymptotically flat, satisfy the dominant energy condition, and have a Cauchy surface $\Sigma$. Let $\alpha^{A}$ be the solution to the Weyl equation whose initial data, on $\Sigma$, satisfies (2.1) and (2.2). Then $\alpha^{A}$ is asymptotically constant at null infinity and $p_{a}$ [defined by (2.6)] is future directed everywhere in $M$.

Even if this conjecture is true, the vector $p_{a}$ will presumably not be unique, since changing the Cauchy surface $\Sigma$ is likely to change $p_{a}$. Nevertheless, the mere existence of such a $p_{a}$ would yield a simple and unified framework for discussing energy in general relativity. For not only would it immediately prove the positivity of the ADM and Bondi energy, it would also prove the decrease of the Bondi energy, and the equality of the past limit of the Bondi energy and the ADM energy.

To the best of our knowledge, this conjecture is open. One small piece of evidence in support of the conjecture comes from the weak field limit. One can show that for perturbations of Minkowski spacetime which satisfy the energy condition, $p_{a}$ is, to leading order, future directed everywhere in $M$.

Of course even if the conjecture turns out to be false, the fact that one can find solutions to the Weyl equation such that $p_{a}$ is future directed on spacelike surfaces 
makes these vectors a useful tool for discussing the relation between local and total energy.

In addition to questions about the total ADM or Bondi energy of a spacetime, these vector fields may play a role in obtaining quasi-local definitions of energymomentum in general relativity. For example, given a spacelike 2-sphere $S$, one can solve Eq. (2.1) on the maximal surface $\Sigma$ spanning $S$ (providing $\Sigma$ exists). Then evolve this initial data via the Weyl equation. One thus obtains a conserved vector field $p_{a}$ in the domain of dependence of $\Sigma$ whose integral is positive and related to the total energy inside $S$. The main problem, however, is to determine what boundary conditions to impose on Eq. (2.1). For finite two-spheres in curved spacetime, constant spinors are not well defined.

Is it possible that a similar expression exists relating local and total angular momentum in general relativity? One possible approach is suggested by the appearance of the twistor equation at null infinity in Sect. 3. Streubel [23] has shown that total angular momentum can be related to two index spinors $\phi_{A B}$ which satisfy the twistor equation at null infinity. It thus seems possible that there exists an analogous relation for angular momentum based on two index spinors.

As we noted in Sect. 2, there is a curious formal similarity between energy in general relativity and charge in electrodynamics : the vector $p^{a}$ is just the chargecurrent vector of the Maxwell field with potential $A_{a}=\alpha_{A} \bar{\alpha}_{A^{\prime}}$. Can this similarity be exploited to further simplify questions in general relativity?

Another way of interpreting the vector $p_{a}$ is in terms of an infinitesimal change in the spacetime connection. One can easily show that if $T_{a b}$ vanishes, then for any vector field $\xi^{a}$ :

$$
p_{a} \xi^{a}=\frac{1}{8 \pi} g^{a b}\left[\mathscr{L}_{K}, \nabla_{a}\right] \xi_{b}
$$

In other words, $p_{a}$ represents the first order change in $\nabla_{a}$ in the direction $K^{a}$.

We believe that the results obtained here shed some light on the origin of Witten's remarkable relation between local and total energy. Of course the deeper mystery still remains: Why is there such a close connection between spinors and gravitational energy?

Acknowledgements. It is a pleasure to thank Roger Penrose and Abhay Ashtekar for useful discussions. We also thank an anonymous referee for pointing out a few errors in the original manuscript.

\section{Appendix}

In this appendix, we consider whether Witten's identity can be used directly to relate $P_{a}^{\mathrm{B}}$ to $T_{a b}$. The crucial question is whether there exist solutions to Eq. (2.1) on an asymptotically null surface $\Sigma^{\prime}$, subject to the boundary conditions (2.14) and (2.15).

It appears to be difficult to show that solutions to this equation exist. The existence theorems which work for the spatial infinity case [10-12] only apply to surfaces $\Sigma$ which are asymptotically Euclidean. This is certainly not the case for $\Sigma^{\prime}$ since asymptotically the metric becomes degenerate. This means that (2.1), while being elliptic everywhere in $\Sigma^{\prime}$ fails to be elliptic at the boundary, which is precisely 
where the data (2.14) and (2.15) is given. Also, the four-dimensional curvature represented by the Riemann tensor falls off only as $O\left(r^{-1}\right)$ near $\mathscr{I}^{+}$in the presence of outgoing radiation.

One indication that solutions do exist is provided by seeking an asymptotic solution expanded in powers of $r^{-1}$. Accordingly we shall now expand (2.1) explicitly in Bondi coordinates using the expansions of [20] for the spin coefficients which appear and show that, order by order, solutions do exist.

The behaviour of the coefficients of the differential operators which appear in the resulting equations depends on the rate at which $\Sigma^{\prime}$ becomes null. For definiteness, we shall suppose that $\Sigma^{\prime}$ is asymptotically like a hyperboloid in Minkowski space, i.e. $\Sigma^{\prime}$ is given by the equation

where

$$
t \equiv u-f(r)=0
$$

$$
f=\frac{1}{r}+O\left(r^{-3}\right) \text {. }
$$

The unit normal to $\Sigma^{\prime}$ takes the form

with $A B=1 / 2$.

$$
t^{a}=A l^{a}+B n^{a}
$$

Taking components of $(2.1)$

$$
\begin{aligned}
& o^{A^{\prime}} D_{A A^{\prime}} \alpha^{A}=0 \\
& l^{A^{\prime}} D_{A A^{\prime}} \alpha^{A}=0
\end{aligned}
$$

with $\alpha^{A}=X O^{A}+Y l^{A}$, we obtain

$$
\begin{gathered}
1 / 2 D X-B^{2} \Delta X-\left(\varrho+B^{2} \gamma\right) X+\bar{\delta} Y-\left(\alpha-\pi / 2+B^{2} v\right) Y=0, \\
\delta X+(\beta-\tau / 2) X-A^{2} D Y+1 / 2 \Delta Y+(\mu-\gamma / 2) Y=0 .
\end{gathered}
$$

The operators $1 / 2 D-B^{2} \Delta$ and $A^{2} D-1 / 2 \Delta$ are related to $\left(\frac{\partial}{\partial r}\right)_{t}$, i.e. differentiation with respect to $r$ in the surface. With the choice (A.2) for $f$ we find

$$
\begin{aligned}
& 1 / 2 D-B^{2} \Delta=\left(1 / 2+O\left(r^{-2}\right)\right) \frac{\partial}{\partial r}+O\left(r^{-4}\right), \\
& A^{2} D-1 / 2 \Delta=r^{2}\left(1 / 2+O\left(r^{-2}\right)\right) \frac{\partial}{\partial r}+O\left(r^{-2}\right) .
\end{aligned}
$$

If we substitute these and the spin coefficients from [20] (after performing the appropriate null rotation) into (A.7) and (A.8), there results

$$
\begin{aligned}
\left(1 / 2+O\left(r^{-2}\right)\right) \frac{\partial X}{\partial r}+\frac{1}{r} X+\frac{1}{r^{3}} \sigma^{0} \bar{\sigma}^{0} X-\frac{1}{r} \bar{\delta} Y \\
+\frac{1}{r^{2}} \bar{\sigma}^{0} \partial Y-\frac{1}{r^{3}} \sigma^{0} \bar{\sigma}^{0} \bar{\partial} Y+\frac{1}{2 r^{2}} ð \bar{\sigma}^{0} Y=O\left(r^{-4}\right), \\
\frac{1}{r} \partial X+r^{2}\left(1 / 2+O\left(r^{-2}\right)\right) \frac{\partial Y}{\partial r}+\frac{1}{r} Y=O\left(r^{-2}\right) .
\end{aligned}
$$


The problem is now to see if asymptotic solutions to these equations exist with the boundary conditions for large $r$ [cf. (3.10) and (3.11)]:

$$
\begin{gathered}
X \rightarrow X_{0}(\theta, \phi), \\
Y \rightarrow Y_{0}(\theta, \phi),
\end{gathered}
$$

where

$$
\begin{aligned}
\bar{\delta} Y_{0}-X_{0} & =0, \\
ð Y_{0} & =0,
\end{aligned}
$$

and

$$
r\left(Y-Y_{0}\right) \rightarrow 0 \text {. }
$$

First consider (A.12). The boundary condition (A.13c) follows immediately from this equation. Furthermore, (A.13a, b) imply that $ð X_{0}+Y_{0}=0$. Imposing this condition, one sees from (A.12) that the stronger condition

$$
r^{3} \frac{\partial Y}{\partial r} \rightarrow 0
$$

must hold. Now consider (A.11). The boundary condition (A.13b) follows from the order $r^{-1}$ part of this equation. The order $r^{-2}$ part of (A.11) determines the next order term in the expansion of $X$. The order $r^{-3}$ part of (A.11), however, is different. Because of (A.14), this order contains no free functions. Hence this is where a contradiction is likely to arise. However, one can see immediately that the $r^{-3}$ part of (A.11) is just $\sigma^{0} \bar{\sigma}^{0}$ times (A.13a). Hence no contradiction occurs. Therefore, at least asymptotically, there exist solutions to (2.1) on an asymptotically null surface $\Sigma^{\prime}$ with the correct boundary conditions. Whether these solutions can be extended to yield a nonsingular solution everywhere on $\Sigma^{\prime}$ is, of course, still an open question.

\section{References}

1. Arnowitt, R., Deser, S., Misner, C.W.: Phys. Rev. 122, 997 (1961)

2. Bondi, H., van der Burg, M.G.J., Metzner, A.W.K.: Proc. R. Soc. (London) A 269, 21 (1962)

3. Sachs, R.K.: Proc. R. Soc. (London) A270, 103 (1962)

4. Ashtekar, A., Magnon-Ashtekar, A.: Phys. Rev. Lett. 43, 181 (1979)

5. Komar, A.: Phys. Rev. 113, 934 (1959)

6. Robinson, D.W., Winicour, J.: Phys. Rev. 3, 840 (1971)

7. Witten, E.: Commun. Math. Phys. 80, 381 (1981)

8. Schoen, R., Yau, S.-T.: Commun. Math. Phys. 65, 45 (1979); 79, 231 (1981)

9. Israel, W., Nester, J.M.: Phys. Lett. 85A, 259 (1981)

10. Choquet-Bruhat, Y., Christodoulou, D.: Acta Math. 146, 129 (1981)

11. Parker, T., Taubes, C.H.: On Witten's proof of the positive energy theorem. Commun. Math. Phys. 84, 223-238 (1982)

12. Reula, O.: Existence theorem for solutions of Witten's Equation and the non-negativity of the total mass. Chicago: University of Chicago preprint

13. Sen, A.: J. Math. Phys. 22, 1781 (1981) 
14. Lichnerowicz, A.: Bull. Soc. Math. France 92, 11 (1964) (Sects. 5 and 22)

15. Nester, J.: Phys. Lett. 83A, 241 (1981)

16. Bramson, B.D.: Proc. R. Soc. (London) A341, 451 (1975)

17. Newman, E.T., Penrose, R.: J. Math. Phys. 3, 566 (1962)

18. Hawking, S.W.: J. Math. Phys. 9, 598 (1968)

19. Newman, E.T., Penrose, R.: J. Math. Phys. 7, 863 (1966)

20. Exton, A., Newman, E.T., Penrose, R.: J. Math. Phys. 10, 1566 (1969)

21. Penrose, R.: Proc. R. Soc. (London) A 284, 159 (1965)

Geroch, R., Horowitz, G.T.: Phys. Rev. Lett. 40, 203 (1978)

22. Penrose, R.: In: Group theory in non-linear problems, Barut, A. (ed.). Boston: D. Reidel 1974

23. Geroch, R., Winicour, J.: J. Math. Phys. 22, 803 (1981)

24. Horowitz, G.T., Perry, M.J.: Phys. Rev. Lett. 48, 371 (1982)

25. Streubel, M.: G. R.G. 9, 551 (1978)

Communicated by A. Jaffe

Received August 2, 1981; in revised form February 22, 1982 
